\title{
The Prevalence of Glaucoma among Pseudoexfoliation Syndrome Patients
}

\author{
Dr. Ali Abdullah Taqi Al-Saffar ${ }^{1 *}$, Dr. Dler Mustafa Ahmed ${ }^{2}$ \\ ${ }^{1}$ Assistant Professor, Ophthalmology Unit, Surgery Department, College of Medicine, University of Sulaimani, Kurdistan-Iraq \\ ${ }^{2}$ Ophthalmologist, Azadi Teaching Hospital, Kirkuk City, Iraq
}

DOI: $10.36348 /$ SJMPS.2019.v05i10.006

| Received: 09.10.2019 | Accepted: 16.10.2019| Published: 21.10.2019

*Corresponding author: Ali A Taqi

Abstract

Introduction: Pseudoexfoliation syndrome is a recognized risk factor for developing glaucoma, cataract and lens dislocation. Pseudoexfoliation syndrome is also associated with increased risk of complications during cataract surgery due to poor mydriasis and zonular weakness. Objective: The aim is to find out the prevalence of glaucoma in Pseudoexfoliation syndrome patients attended to Shaheed Doctor Aso hospital, Sulaimaniya city, Kurdistan region of Iraq and Azadi Teaching Hospital, Kirkuk city - Iraq. Patients and Methods: This cross sectional study encompassed 378 patients (756 eyes) with whom the pseudoexfoliation syndrome was detected as an incidental finding aged 50 years or older. A detailed evaluation including general and ophthalmic history, slit lamp biomicroscopy, intraocular pressure measurement (TOPCON computerized tonometer CT-80 and Goldmann Applanation tonometer), gonioscopy (Goldmann three mirrors goniolens) and dilated eye examination were performed. Oral informed consent was obtained from all participants. Results: Out of the 378 patients who had pseudoexfoliation syndrome, 58 (15.3\%) were diagnosed with pseudoexfoliation glaucoma and 34 patients $(9 \%)$ with ocular hypertension. In the glaucomatous patients, men to women ratio was $2: 1$, this was statistically significant $(p=0.001)$. Most of patients in the age group $(70-79)$ years of age, represent 23.3\%. Bilateral Pseudoexfoliation syndrome was associated more with pseudoexfoliation glaucoma (58.6\%), while unilateral pseudoexfoliation syndrome was associated more with ocular hypertension (64.7\%). The Kurdish race patients expressed the highest prevalence of pseudoexfoliation glaucoma and ocular hypertension (31.5\%) followed by the Arabic race $(20 \%)$ and lastly the Turkmen (16\%). Conclusion: Pseudoexfoliation syndrome showed to be a significant glaucoma risk factor locally, with a prevalence of (15.3\%), and if we added the Ocular hypertension (9\%), it will be (24.3\%). Recommendation: Patients with Pseudoexfoliation syndrome should be informed and examined at least annually as the risk may be present throughout their life and increasing with age.

Key words: Intraocular pressure, Pseudoexfoliation syndrome, Glaucoma, Ocular hypertension, Goldmann Applanation tonometer.

Copyright @ 2019: This is an open-access article distributed under the terms of the Creative Commons Attribution license which permits unrestricted use, distribution, and reproduction in any medium for non-commercial use (NonCommercial, or CC-BY-NC) provided the original author and source are credited.

\section{INTRODUCTION}

Pseudoexfoliation syndrome (PXS) is an important ocular manifestation of a systemic disorder and the most identifiable cause of secondary open-angle glaucoma. Because of the insidious and asymmetrical nature of this disease, PXS glaucoma is often far advanced when diagnosed. Markedly elevated intraocular pressure (IOP), most often with an open angle, is common in this syndrome. Although not all patients with PXS will develop glaucoma, there is no reliable way to predict which patients with PXS syndrome will develop optic nerve damage and vision loss [1].
The pseudoexfoliation syndrome (PXS) is characterized by the accumulation of dandruff like fluffy deposits of fibrillar granular material. Pseudoexfoliative material is a grey-white material of a microfibrillar core surrounded by an amorphous matrix. It is composed of amyloid-like material; and may be derived from abnormal extracellular matrix metabolism in ocular and other tissues. The material is deposited on various ocular structures including the lens capsule, zonular fibers, iris, trabeculum and conjunctiva. Pseudoexfoliative material has been found in skin and visceral organs, leading to the concept of PXS as the ocular manifestation of a systemic disorder; PXS is associated with an increased prevalence of vascular disorders, hearing loss and Alzheimer disease. Plasma homocysteine tends to be higher than controls, and 
inadequate dietary folate intake (folate reduces homocysteine) may be a risk factor [2].

Mutations in a single gene, lysyl oxidase-like protein 1 (LOXL1) gene, seem to be present in nearly all cases of PXS syndrome and PXS glaucoma; however, these disease-associated mutations are also common in the population without glaucoma, suggesting that the disorder is multifactorial. The exact mechanism by which LOXLl mutations are related to the development of glaucoma is unclear, but it likely involves the reduced or abnormal synthesis of elastin fibers [3].

Diagnostic features of PXS include deposition of PXS material, endothelial pigmentation, loss of pupillary ruff, iris sphincter transillumination, Sampaolesi line, and pigment deposition in the trabecular meshwork. Pseudoexfoliation syndrome is associated with various ocular complications including elevated intraocular pressure, glaucoma, poor mydriasis, zonular weakness, corneal endotheliopathy, and higher rate of vitreous loss during cataract surgery, postoperative complications such as capsular phimosis, and opacification. However, in most cases PXS remains asymptomatic [2].

Exfoliation syndrome has been associated with glaucoma since first reported by Lindberg in $1917.1 \mathrm{He}$ found the characteristic white dandruff-like flakes on the lens and pupillary margin in 50\% of his patients with glaucoma. Later authors have confirmed and expanded on Lindberg's report. Vogt attributed the material to a true exfoliation or delamination of the lens capsule, whereas Busacca and years later DvorakTheobald attributed the material to an abnormal precipitation on the lens capsule. Dvorak-Theobald called it pseudoexfoliation to distinguish it from true exfoliation and delamination of the lens capsule as seen in glass blowers [4].

Many studies carried out on IOP used the GAT which is indeed the gold standard to measure IOP. In this study, we used a noncontact (air puff) tonometer to measure the IOP of the included cases. This tonometer is based on the same physical principle which is used for GAT, i.e., the Imbert-Fick principle, which states that the force required to flatten a spherical container (F) equals the product of the internal pressure $(\mathrm{P})$ by the area of flattening $(\mathrm{A})$; thus $\mathrm{F}=\mathrm{P} \times \mathrm{A}$. This force depends on several factors, such as the nature of the tear film and corneal curvature, and is also subject to the influence of CCT. The non-contact tonometer eliminates the need for corneal contact and topical anesthesia, thereby avoiding the potential problems of corneal abrasion and spread of infection. In addition, a recent comparison by Shepens et al. of the two types of tonometers demonstrated that there was no statistically significant difference between values given by each tonometer for the same patient [5].

Data on the clinical profile and prevalence of PXS is important due to the fact that the world population is aging, and the proportion of elderly is increasing globally. Exfoliation syndrome occurs worldwide, although reported prevalence rates vary extensively. Reasons for this variation reflect a combination of true differences in prevalence on the basis of racial, ethnic, the age and gender distribution of the patient cohort or population group examined; the clinical criteria used for making a diagnosis of exfoliation syndrome; the ability of the examiner to detect early stages and/or more subtle manifestations of the disorder; and the thoroughness of examination, or other as yet unknown reasons. In particular, many cases go undetected because of failure to dilate the pupil, or to examine the lens with the slit lamp after dilation, and because of a low index of suspicion on the part of the examiner [6].

\section{Aim}

The aim is to find out the prevalence of glaucoma in PXS syndrome patients and to determine patient's characteristics.

\section{Patients and methods}

This was a cross sectional study conducted from January 2016 to June 2016 in Shaheed Dr.Aso hospital, Sulaimani city, Kurdistan region of Iraq and Azadi Teaching Hospital, Kirkuk city - Iraq.

The study was conducted in accordance with a protocol approved by the scientific and ethics committee at Sulaimani University. Moreover, the oral informed consent was obtained from all individuals and is included in the study.

Three hundred and seventy eight (378) participants (seven hundred and fifty six 756 eyes), in whom pseudoexfoliation syndrome was detected as an incidental finding during a routine ophthalmologic examination form the population of this study. The patients were aged fifty years and older, and divided into 4 age groups $(50-59,60-69,70-79,80-89)$ years.

\section{Exclusion criteria}

The following criteria are excluded in the patients:

- Age less than 50 years.

- Have history of glaucoma.

- Using systemic drugs that may lower IOP (most importantly beta blockers).

- Has undergone complicated ocular surgery.

- Has history of trauma.

- Has corneal epithelial defect, epithelial or stromal edema, corneal opacities, dystrophies, degenerations or ectasias. 
- Signs of ongoing or previous intraocular inflammation.

A prepared form inquiring participant's name, gender, age group, ethnicity, residence, systemic illness, past ophthalmic history, slit-lamp examination for presence of PXS and phakic state, IOP, visual field, Gonioscopy, and CD ratio was filled. Name of the participants was not a mandatory information, and a code number was given to each participant.

Participants underwent a medical, family, and ocular history with a comprehensive bilateral ophthalmological examination, including external and slit-lamp examination, measurement of intraocular pressure, and indirect ophthalmoscopy were done on each patient using an indirect ophthalmoscopy lens (Volk 90) around 20 minutes to half an hour after instillation of $0.5 \%$ mydriacyl ${ }^{\circledR}$ (Alcon). Gonioscopy using Goldmann three mirror goniolens and Visual fields clinically.

The diagnosis of exfoliation syndrome was made on the basis of the presence of "dandruff-like" material on the pupillary margin of iris and the anterior lens capsule which typically shows a central disc and a radially indented peripheral layer of PXS material, separated by a clear zone maintained by pupillary rubbing. In the majority of patients the diagnosis was made prior to dilatation, and in all patients the diagnosis was confirmed by examination after dilatation.

IOP measurement was done using an air-puff tonometer (TOPCON computerized tonometer CT-80, Japan, mean of three successful measurements) by an experienced-hand medical staff. The IOP measurement was remeasured if it was $22 \mathrm{~mm} \mathrm{Hg}$ or more with the Goldmann Applanation tonometer (GAT). GAT done after topical anaesthetic with Alcaine ${ }^{\circledR}$ (Alcon) instillation, drying of excessive drop that overflows onto the lower lid and waiting for around 30 seconds to 2 minutes, and a small amount of fluorescein was instilled into the conjunctival sac. The patient was positioned at the slit lamp with his or her forehead firmly against the headrest and instructed to look straight ahead and to breathe normally. With the cobalt blue filter in place and illumination of maximal intensity directed obliquely (approximately $60^{\circ}$ ) at the prism, the prism was centered in front of the apex of the cornea. The dial was preset at 1 (i.e. $10 \mathrm{mmHg}$ ) and was advanced until it just touched the apex of the cornea and the reading was recorded.

Many patients find the anticipation of multiple puffs uncomfortable, which can lead to false high pressure measurements because of lid pressure. For such patients the use of topical anaesthetic drop can eliminate this discomfort. In this study we made further assessment of IOP when the air puff tonometer estimated greater than normal readings, as the noncontact tonometer is less reliable in patients with elevated intraocular pressure.

\section{Measurement of IOP was recorded as}

- Normal.

- Ocular hypertension.

- Glaucoma.

Eyes that had an IOP less than $21 \mathrm{~mm} \mathrm{Hg}$ were considered as normal, while those that had an IOP greater than $22 \mathrm{~mm} \mathrm{Hg}$ but showed normal visual fields and discs are considered as having ocular hypertension. The diagnosis of glaucoma was made in eyes that have an IOP greater than $22 \mathrm{~mm} \mathrm{Hg}$ and glaucomatous cupping and/or a visual field test results after requesting for a visual field test if necessary. Humphrey Field Analyzer (Ziess) screening program was used for this purpose. Glaucoma specific visual field defects (after other possible causes for the visual field findings were excluded) included: nasal steps, paracentral defects, arcuate defects, central islands, temporal islands, and altitudinal loss. Compatible glaucomatous optic nerve damage included asymmetric cupping with an interocular difference of 0.2 or more; cup to disc ratio of 0.7 or more and limited loss of neural rim tissue to disc margin, including notching of neural rim tissue, diffuse thinning of neural rim, disc or peripapillary nerve fiber layer hemorrhage, and thinning or defect in the nerve fiber layer. IOP was not considered alone in the diagnosis of glaucoma.

Indirect gonioscopy was done as a method of evaluating the AC angle, by using Goldmann three mirrors goniolens. The angle was examined for the presence of patchy trabecular and Schwalbe line hyperpigmentation especially inferiorly, Sampaolesi line: an irregular band of pigment running on or anterior to the Schwalbe line, Dandruff-like pseudoexfoliated materials deposits in the angle.

The angle was examined for patency and graded by using Shaffer system which records the angle in degrees between two imaginary lines tangential to the inner surface of the trabeculum and the anterior surface of the iris about one-third of the distance from its periphery.

Patients' Data forms were checked for any error or inconsistency then transferred into a computerized database program; SPSS (statistical package for social sciences) software version 23 for windows was used in statistical analysis. All variables were coded with a specific code for each variable and prepared for statistical analysis.

Descriptive statistics were presented as frequency (number of cases) with proportions (percentages) for categories. 
The Pearson's chi square test was used to test: (a) the significance of association between aging and the occurrence of PXG, (b) the difference in prevalence of glaucoma between men and women and (c) the comparison between the unilaterality and bilaterality of the involved PXS patents with IOP status.

In all statistical tests and procedures, level of significance ( $\mathrm{P}$. value) was set at $\mathrm{P} \leq 0.05$ considered as significant difference or association.

Finally, the results were presented in tables and figures.

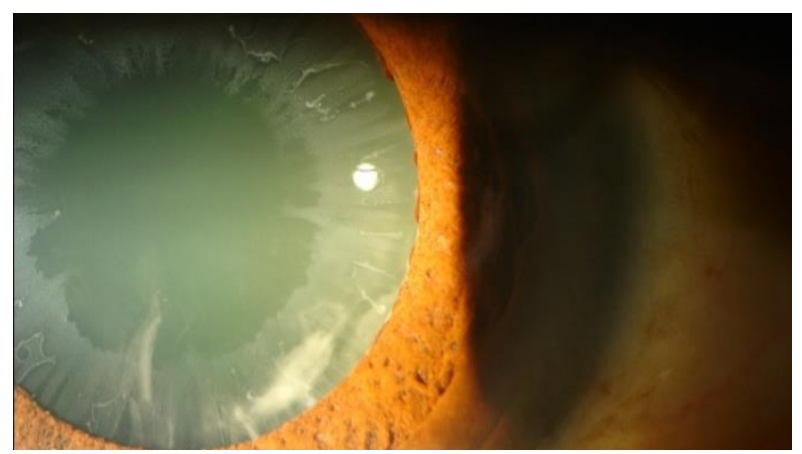

Fig-1: An eye with PXS showing the dandruff like pseudoexfoliation material on the surface of the cataractous lens capsule

\section{RESULTS}

Out of the 378 patients who had PXS syndrome, $58(15.3 \%)$ were diagnosed with pseudoexfoliation glaucoma and 34 patients (9\%) with $\mathrm{OH}$.

There were $176(46.6 \%)$ males and 202 $(53.4 \%)$ females. The ethnical distribution of the included patients was as the following: Kurds 178 cases (47.1\%), Arabs 100 cases (26.5\%) and Turkman 100 cases $(26.5 \%)$. According to their residency, there were 99 patients ( $26.2 \%$ ) from inside Sulaimani city while 279 patients $(73.8 \%)$ from outside Sulaimani city including participants from Kirkuk city. These are shown in detail in table 1.

Table-1: Demographic characteristics of the involved patients.

\begin{tabular}{|c|c|c|c|}
\hline \multicolumn{2}{|c|}{} & Frequency & Percent \\
\hline \multirow{3}{*}{ Gender } & Male & 176 & $46.6 \%$ \\
\cline { 2 - 4 } & Female & 202 & $53.4 \%$ \\
\hline \multirow{2}{*}{ Residency } & Urban & 101 & $26.7 \%$ \\
\cline { 2 - 4 } & Rural & 279 & $73.3 \%$ \\
\hline \multirow{2}{*}{ Ethnicity } & Kurds & 178 & $47.1 \%$ \\
\cline { 2 - 4 } & Arabs & 100 & $26.5 \%$ \\
\cline { 2 - 4 } & Turkman & 100 & $26.5 \%$ \\
\hline
\end{tabular}

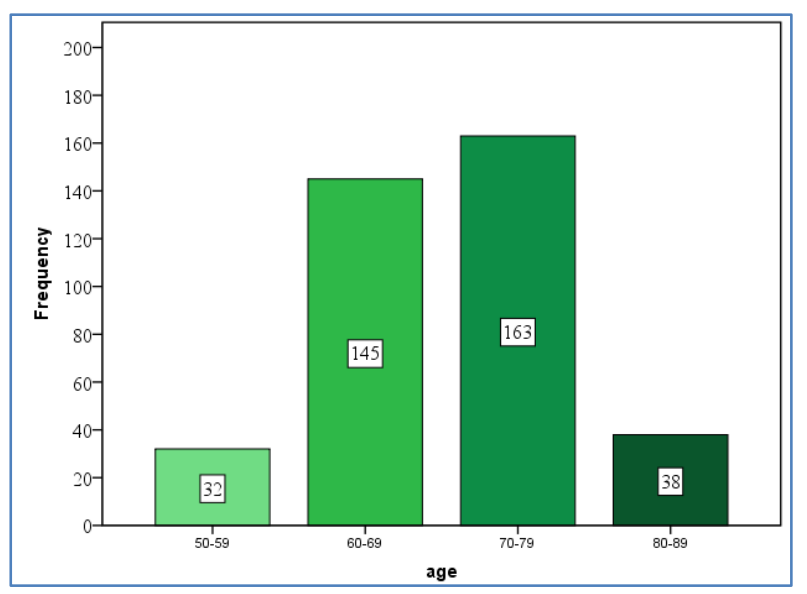

Fig-2: The age groups of the population of the study

The patients were divided into four age groups and the number of patients in each group is demonstrated in as seen in figure 1 :

The frequency of $\mathrm{OH}$ and $\mathrm{PXG}$ among the age groups of the patients in this study is considered in table 2 .The prevalence of PXG increased with age and the association between glaucoma and age was statistically significant $(p=0.001)$.

Table-2: Age distribution of the involved patients

\begin{tabular}{|l|l|l|l|l|l|l|}
\hline & \multicolumn{5}{|c|}{ IOP status } & \multirow{2}{*}{ P value* } \\
\hline & & Normal & OH & PXG & total & \\
\hline \multirow{3}{*}{$\begin{array}{l}\text { Age } \\
\text { groups }\end{array}$} & $50-59$ & $22(68.8 \%)$ & $4(12.5 \%)$ & $6(18.8 \%)$ & 32 & \multirow{2}{*}{0.001} \\
\cline { 2 - 6 } & $60-69$ & $113(77.9 \%)$ & $20(13.8 \%)$ & $12(8.3 \%)$ & 145 & \\
\cline { 2 - 6 } & $70-79$ & $115(70.6 \%)$ & $10(6.1 \%)$ & $38(23.3 \%)$ & 163 & \\
\cline { 2 - 6 } & $80 \geq$ & $36(94.7 \%)$ & $0(0 \%)$ & $2(5.3 \%)$ & 38 & \\
\cline { 2 - 6 } & Total & $286(75.7 \%)$ & $34(9.0 \%)$ & $58(15.3 \%)$ & 378 & \\
\hline
\end{tabular}

IOP characteristics of PXS patients are given in figure 2. Two hundred and eighty six patients 286 (75.7\%) had a normal IOP, thirty four $34(9.0 \%)$ had ocular hypertension, and fifty eight 58 patients (15.3\%) had glaucoma. 


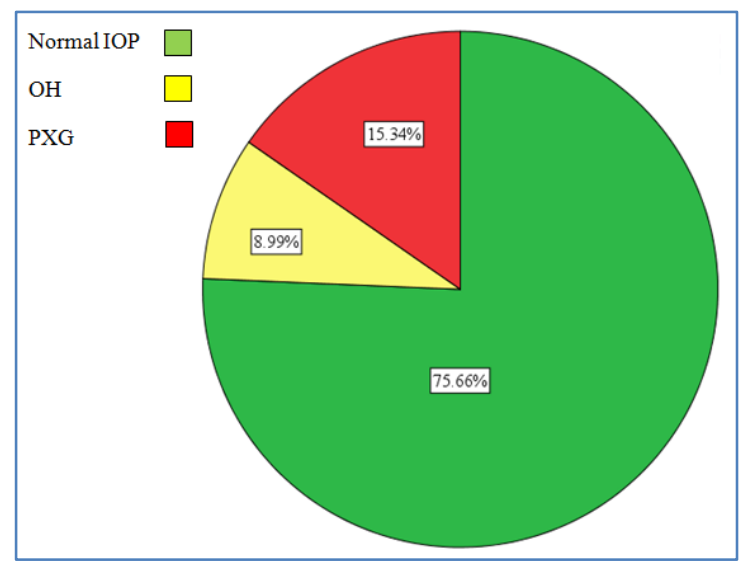

Fig-3: The IOP characteristics of the population of the study

In comparing the unilaterality and bilaterality of the involved PXS patents with IOP status, there was 22 patients $(64.7 \%)$ that had unilateral $\mathrm{OH}$, while 12 patients $(35.3 \%)$ has bilateral $\mathrm{OH}$, and the number of PXS patients with unilateral glaucoma was 24 patients $(41.4 \%)$, while bilateral involvement with glaucoma was found in 34 patients $(58.6 \%)$ as shown in table 3 which correlates 1OP status in the unilaterally and bilaterally involved groups. We found no statistically significant association between $\mathrm{OH}$ or glaucoma and the unilaterality and bilaterality of the PXS syndrome ( $p$ $=0.55$ ).

Table 4 correlates ethnicity and IOP status. For each group ocular hypertension and glaucoma occur at a slightly higher prevalence in the Kurds $(31.5 \%)$ than in Arabic ethnicity (20\%) or Turkman (16\%):

Table-3: IOP Characteristics Relative to Patients Ethnicity

\begin{tabular}{|l|l|c|c|c|c|}
\hline & \multicolumn{5}{|c|}{ I.O.P status } \\
\hline & & Normal IOP & OH & PXG & total \\
\hline \multirow{3}{*}{ Ethnicity } & Kurds & $122(68.5 \%)$ & $16(9.0 \%)$ & $40(22.5 \%)$ & 178 \\
\cline { 2 - 6 } & Arabs & $80(80.0 \%)$ & $10(10.0 \%)$ & $10(10.0 \%)$ & 100 \\
\cline { 2 - 6 } & Turkman & $84(84.0 \%)$ & $8(8.0 \%)$ & $8(8.0 \%)$ & 100 \\
\cline { 2 - 6 } & Total & $286(75.7 \%)$ & $34(9.0 \%)$ & $58(15.3 \%)$ & 378 \\
\hline
\end{tabular}

The gender and the IOP status were demonstrated in table 5, where it is obvious that the percentage of PXS males with abnormal IOP: glaucoma and $\mathrm{OH}(34.1 \%)$ was more than the female ones $(15.8 \%)$. The association between PXG and gender was statistically significant $(p=0.001)$.

Table-4: Gender - IOP State Crosstabulation

\begin{tabular}{|l|l|c|c|c|c|c|}
\hline & \multicolumn{5}{|c|}{ I.O.P status } & \multirow{2}{*}{ P value } \\
\hline & & Normal IOP & OH & PXG & Total & \\
\hline \multirow{2}{*}{ Gender } & Male & $116(65.9 \%)$ & $22(12.5 \%)$ & $38(21.6 \%)$ & 176 & \multirow{2}{*}{0.001} \\
\cline { 2 - 7 } & Female & $170(84.2 \%)$ & $12(5.9 \%)$ & $20(9.9 \%)$ & 202 & \\
\hline
\end{tabular}




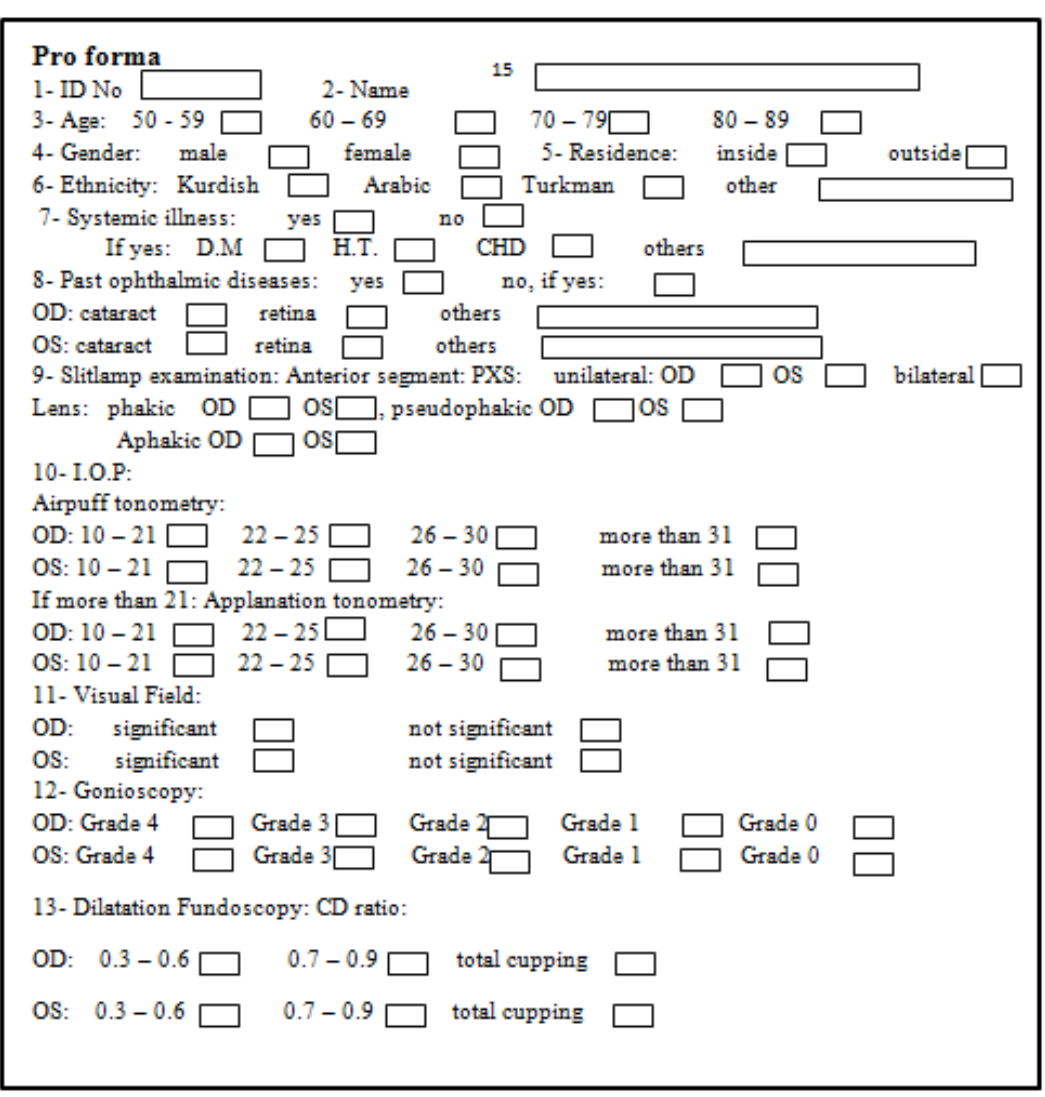

\section{DISCUSSION}

The result of this study which showed a prevalence of $15.3 \%$ of eyes with PXS had glaucoma reflects a comparable prevalence to that reported by Arvind et al. [7] and the Blue Mountains Eye Study[8], which found prevalences of $16.7 \%$ and $14.2 \%$, respectively. However, a hospital-based study in Saudi Arabia and Pakistan found $45 \%$ and $40 \%$ of patients with PXS to have high IOP [6,9]. Also the prevalence of elevated IOP associated with exfoliation syndrome in this study was lower than has been reported by Al Jaffal $\mathrm{Z}$ and Kayyali in Syria (37.5\%)[10], Al-Bdour et al. in Jordan (33.1\%),[11] Shazly et al. in Egypt (30.4\%)[12] and Yalaz et al. in Turkey (32.1\%)[13]. In India, 34\% of exfoliation syndrome patients have "manifest glaucoma," although the criteria for the diagnosis of glaucoma are not detailed[14].

The association of glaucoma and exfoliation syndrome has been reported to be from $0 \%$ to $100 \%$. Factors influencing this wide variation in results include methods of patient selection, thoroughness of examination, definition of "glaucoma," and, possibly, variability due to ethical considerations [15].

In analyzing published data pertaining to the association of glaucoma and exfoliation syndrome, it is important to differentiate between data reporting the frequency of exfoliation syndrome in known glaucoma patients and data reporting the frequency of glaucoma in known exfoliation syndrome patients. The literature is replete with reports concerning the former, but reports concerning the latter are scant. Of the few studies reporting the frequency of glaucoma in unselected patients who have exfoliation syndrome, none in the recent literature has been reported in patients in Iraq.

In Greece the prevalence of PXG among PXS patients was $22.1 \%$ [16], which is also higher than the result of this study. In Norway, the frequency of glaucoma in PXS patients was $23 \%$, in which the diagnosis of glaucoma included patients in whom the discs and visual field were normal but who had elevated 1OP, which was designated as having ocular hypertension in this study. Because the number of patients who have glaucomatous cupping and field loss is not mentioned, a direct comparison with data from the previous study is not possible. However, in terms of exfoliation syndrome patients with IOP abnormalities; the results in the present study are almost identical to those reported in Aasvad's study [17].

A strong relationship between glaucoma and PXS is known [18]. Subjects with PXS had a two- to three fold increased risk for glaucoma according to the Blue Mountains Eye Study [8]. Moreover, Topouzis et al. reported increased likelihood of glaucoma at the same IOP in subjects with PXS [19].

This extensive variation in the reported prevalence of PXG among PXS patients may reflect a 
combination of true differences due to racial, ethnic, or other as-yet-unknown reasons; the age and sex distribution of the patients or population group examined; the clinical criteria used to diagnose PXS; the ability of the examiner to detect early stages; the thoroughness of the examination; and the awareness of the observer [20].

Within the bilateral and unilateral groups of the PXS patients, the diagnosis of glaucoma and ocular hypertension is made at different rates. $\mathrm{OH}$ is more prevalent $(64.7 \%)$ in the unilateral PXS patients, while $58.6 \%$ of the PXG patients where affected bilaterally. In this study, there was no statistically significant association between $\mathrm{OH}$ or glaucoma and the unilaterality and bilaterality of the PXS syndrome ( $p=$ 0.55). Significant differences in clinical presentation and asymmetry of clinical findings and glaucomatous disease between two eyes of a patient is common. Careful examination with the slit lamp is essential for diagnosis or for at least raising suspicion of the diagnosis in the setting of subtle or early findings. Not uncommonly, the clinical findings are only evident in one eye, even though this is a systemic disorder. Although the PXS material may be seen bilaterally, it is more often noted to be unilateral. However, in most 'unilateral' cases the apparently uninvolved eye has subclinical PXS syndrome, as multiple electron microscopy studies demonstrate. Therefore, asymmetrical may be a better term to use than unilateral when describing this entity [1]. In general, patients with bilateral involvement tend to be older [21].

Various ethnic patients included in this study showed different prevalences of abnormal IOP status. The Kurdish nationality expressed the highest prevalence of PXG and $\mathrm{OH}(31.5 \%)$ followed by the Arabic (20\%) and lastly the Turkman (16\%). This variability in the prevalence of PXG may be primarily related to the different prevalence of PXS in the different ethnic groups. There is extensive variation in the prevalence of PXS in different populations, which may be related to variations in genetic, geographical or racial factors or may simply reflect differences in study design or sampling methods [11]. In some populations almost all patients with PXS have certain single nucleotide polymorphisms (SNPs) in the LOXL1 gene, which is involved in elastin fiber production [2]. Genetic factors are now considered as predisposing factors for PXS, although results are not clear and studies are still ongoing [22].

Geographical factors are also important in the context of PXS. Stein et al. reported an increased PXS frequency with increase in the annual number of sunny days [23]. Sufi et al. showed increased prevalence of PXS in people exposed to outdoor activity. Majority of people in rural Kashmir depend on agriculture as source of income and thus would be exposed to outdoor activities [24]. This supports the association between environmental factors (possibly solar radiation, UV exposure) and PXS as documented by other studies [25], and generally the agricultural life style of the Kurdish and the Arabic people may explain the increased prevalence of the PXS among them, although we were not able to examine this theory, as we did not collect detailed data on sunlight exposure.

In both the unilaterally and bilaterally involved populations, the percentage of women with nonglaucomatous eyes was greater than the percentage of men. The association between PXG and gender was statistically significant in the present study $(p=0.001)$. The finding of a preponderance of women in the PXS population without glaucoma reported in this study confirms previous reports by Aasved [20], Hansen [26] and Tarkkanen[15]. This study revealed higher rates of elevated IOP among male $(34.1 \%)$ than female patients $(15.8 \%)$, which is in contrast to other studies that revealed no sex predilection in exfoliation syndrome eyes that have glaucoma [27], or more female preponderance [28]. The reason for this is not known [29].

In this study, increasing age was associated with increase in prevalence of PXG. This may be caused by increasing the prevalence of PXS with advancing age. The age distribution of exfoliation syndrome patients reported in this study is similar to that of previous reports $[8,30]$. It was clear that the association between glaucoma and advancing age was statistically significant $(p=0.001)$. Although the reason for this age-related increase is unknown, it has been speculated that the changes in gene expression that occur with age may be responsible [24].

\section{Limitation}

One of the limitations of this study is being a hospital-based rather than a population-based study. Over or under-estimation of the prevalence of PXS and or co-morbidities associated with PXS may be attributed to the hospital based nature of the study. Another limitation of this study is that it is a study of people at one fraction of time to determine whether PXS syndrome is associated with the occurrence of $\mathrm{OH}$ and glaucoma (cross-sectional study). Because the disease $(\mathrm{OH}, \mathrm{PXG})$ outcome and the possible risk factor (PXS syndrome) are measured at the same time, the study provides a 'snapshot' view of their relationship. PXS and glaucoma-OH have already occurred and it is not possible to be sure when it happened first. So, the study cannot provide accurate information about causality.

Many major questions about PXS syndrome still remain obscure. The variation of its prevalence in different populations, its pathogenesis, and the 
composition of extraocular deposits are some of the topics that have to be clarified.

\section{CONCLUSION}

\section{This study concludes that}

- At the time of initial diagnosis of PXS syndrome, $15.3 \%$ of patients had glaucoma, $9 \%$ of patients had ocular hypertension.

- In exfoliation syndrome with glaucoma, male affected more than female

- Greater percentage of patients with bilateral exfoliation syndrome has glaucoma, as compared with patients having unilateral exfoliation syndrome.

- Greater percentage of patients with unilateral exfoliation syndrome has $\mathrm{OH}$, as compared with patients having bilateral exfoliation syndrome.

\section{RECOMMENDATIONS}

- Ophthalmologists in Iraq should focus on the detection of PXS and to check IOP in PXS patients, especially considering the risks for operative complications related to PXS and the higher prevalence of glaucoma among PXS patients.

- We recommend a larger prospective study looking at the prevalence of PXS in the local community would be helpful to decipher the unique epidemiology of PXS in our local population.

- PXS constitutes a major glaucoma risk factor; patients with PXS syndrome should be informed and examined annually as the risk may be present throughout.

Acknowledgment: Thanks to Shaheed Dr. Aso hospital and Azadi teaching hospital, the staff, and managers.

\section{Financial Disclosure: None}

\section{Conflict of interest: None}

\section{REFERENCES}

1. Yanoff, M., \& Duker, J. S. (2014). Ophthalmology. Mosby, 4th ed.: 1070.

2. Bowling, B., Kanaski Clinical Ophthalmology. (2016). ELSEVIER, 8th ed.: 367-368.

3. Amith, H. V., Ankola, A. V., \& Nagesh, L. (2011). Lip prints-can it aid in individual identification. $J$ Oral Health Comm Dent, 5(3), 113-8.

4. Albert, D. M., \& Jakobiec, F. A. (Eds.). (2000). Principles and practice of ophthalmology (Vol. 1). WB Saunders Company.

5. Schepens, G., Urier, N., Bechetoille, A., \& De Potter, P. (2001). Correlation entre tonometrie de Goldmann et tonometrie non-contact en fonction de l'epaisseur corneenne. Bulletin de la Société belge d'ophtalmologie, (279), 15.

6. Al-Saleh, S. A., Al-Dabbagh, N. M., Al-Shamrani, S. M., Khan, N. M., Arfin, M., Tariq, M., \& AlFaleh, H. M. (2015). Prevalence of ocular pseudoexfoliation syndrome and associated complications in Riyadh, Saudi Arabia. Saudi medical journal, 36(1), 108.

7. Arvind, H., Raju, P., Paul, P. G., Baskaran, M., Ramesh, S. V., George, R. J., ... \& Vijaya, L. (2003). Pseudoexfoliation in south India. British Journal of Ophthalmology, 87(11), 1321-1323.

8. Mitchell, P., Wang, J. J., \& Hourihan, F. (1999). The relationship between glaucoma and pseudoexfoliation: the Blue Mountains Eye Study. Archives of Ophthalmology, 117(10), 13191324.

9. Rao, R. Q., Arain, T. M., \& Ahad, M. A. (2006). The prevalence of pseudoexfoliation syndrome in Pakistan. Hospital based study. BMC ophthalmology, 6(1), 27.

10. Zuhair Al Jaffal, M. D., \& Kayyali, A. (2015). ليص . Journal of the Arab Board of Health Specializations Vol, 16(4).

11. Al-Bdour, M. D., Al-Till, M. I., Idrees, G. M., \& Abu Samra, K. M. (2008). Pseudoexfoliation syndrome at Jordan University Hospital. Acta ophthalmologica, 86(7), 755-757.

12. Shazly, T. A., Farrag, A. N., Kamel, A., \& AlHussaini, A. K. (2011). Prevalence of pseudoexfoliation syndrome and pseudoexfoliation glaucoma in Upper Egypt. BMC ophthalmology, 11(1), 18.

13. Yalaz, M., Othman, I., Nas, K., Eroĝlu, A., Homurlu, D., Çikintas, Z., \& Ashouri, A. (1992). The frequency of pseudoexfoliation syndrome in the eastern Mediterranean area of Turkey. Acta ophthalmologica, 70(2), 209-213.

14. Sood, N.N., Ratnaraj, A.(1968). Pseudoexfoliation of the lens capsule. Orient Arch Ophthal; 6:62-7.

15. Tarkkanen, A. (1962). Pseudoexfoliation of the lens capsule. A clinical study of 418 patients with special reference to glaucoma, cataract, and changes of the vitreous. Acta ophthalmologica. Supplementum, 1.

16. Andrikopoulos, G. K., Mela, E. K., Georgakopoulos, C. D., Papadopoulos, G. E., Damelou, A. N., Alexopoulos, D. K., \& Gartaganis, S. P. (2009). Pseudoexfoliation syndrome prevalence in Greek patients with cataract and its association to glaucoma and coronary artery disease. Eye, 23(2), 442.

17. AASVED, H. (1971). Intraocular pressure in eyes with and without fibrillopathia epitheliocapsularis: (so-called senile exfoliation or pseudoexfoliation). Acta ophthalmologica, 49(4), 601-610.

18. Henry, J. C., Krupin, T., Schmitt, M., Lauffer, J., Miller, E., Ewing, M. Q., \& Scheie, H. G. (1987). Long-term follow-up of pseudoexfoliation and the development of elevated intraocular pressure. Ophthalmology, 94(5), 545-552.

19. Topouzis, F., Harris, A., Wilson, M. R., Koskosas, A., Founti, P., Yu, F., ... \& Coleman, A. L. (2009). Increased likelihood of glaucoma at the same 
screening intraocular pressure in subjects with pseudoexfoliation: the Thessaloniki Eye Study. American journal of ophthalmology, 148(4), 606-613.

20. AASVED, H. (1969). THE GEOGRAPHICAL DISTRIBUTION OF FIBRILLOPATHIA EPITHELIOCAPSULARIS: so-called senil exfoliation or Pseudoexfoliation of the anterior lens capsule. Acta ophthalmologica, 47(3), 792-810.

21. Hiller, R., Sperduto, R. D., \& Krueger, D. E. (1982). Pseudoexfoliation, intraocular pressure, and senile lens changes in a population-based survey. Archives of Ophthalmology, 100(7), 10801082.

22. Pohjanpelta P. E. J., Hurskainen, L.(1972). Studies on relatives of patients with glaucoma simplex and patients with pseudoexfoliation of the lens capsule. Acta Ophthalmologica, 50(2):255-261.

23. Stein, J. D., Pasquale, L. R., Talwar, N., Kim, D. S., Reed, D. M., Nan, B., ... \& Richards, J. E. (2011). Geographic and climatic factors associated with exfoliation syndrome. Archives of ophthalmology, 129(8), 1053-1060.

24. Sufi, A. R., Mufti, A. A., Nazir, N., Qureshi, T., \& Ramzan, R. (2014). Prevalence of pseudoexfoliation syndrome in patients scheduled for cataract surgery in eye camps in
Kashmir. Journal of Clinical Ophthalmology and Research, 2(3), 137.

25. Taylor, H. R. (1980). The environment and the lens. British Journal of Ophthalmology, 64(5), 303310.

26. Hansen, E., \& Sellevold, O. J. (1968). Pseudoexfoliation of the lens capsule I. Clinical evaluation with special regard to the presence of glaucoma. Acta ophthalmologica, 46(6), 1095 1104.

27. HØRVEN, I. (1966). Exfoliation syndrome: incidence and prognosis of glaucoma capsulare in Massachusetts. Archives of Ophthalmology, 76(4), 505-511.

28. Karger, R. A., Jeng, S. M., Johnson, D. H., Hodge, D. O., \& Good, M. S. (2003). Estimated incidence of pseudoexfoliation syndrome and pseudoexfoliation glaucoma in Olmsted County, Minnesota. Journal of glaucoma, 12(3), 193-197.

29. Kozart, D. M., \& Yanoff, M. (1982). Intraocular pressure status in 100 consecutive patients with exfoliation syndrome. Ophthalmology, 89(3), 214218.

30. ODLAND, M., \& AASVED, H. (1973). Follow-up of initially nonglaucomatous patients with fibrillopathia epitheliocapsularis (so-called senile exfoliation of the anterior lens capsule). Acta Ophthalmologica, 51(S120), 77-81. 